\title{
Review Article \\ Do LINEs Have a Role in X-Chromosome Inactivation?
}

\author{
Mary F. Lyon \\ Mammalian Genetics Unit, Medical Research Council (MRC), Harwell, Oxfordshire OX11 ORD, UK
}

Received 31 May 2005; Revised 22 November 2005; Accepted 4 December 2005

\begin{abstract}
There is longstanding evidence that X-chromosome inactivation (XCI) travels less successfully in autosomal than in X-chromosomal chromatin. The interspersed repeat elements LINE1s (L1s) have been suggested as candidates for "boosters" which promote the spread of XCI in the X-chromosome. The present paper reviews the current evidence concerning the possible role of L1s in XCI. Recent evidence, accruing from the human genome sequencing project and other sources, confirms that mammalian $\mathrm{X}$-chromosomes are indeed rich in L1s, except in regions where there are many genes escaping XCI. The density of L1s is the highest in the evolutionarily oldest regions. Recent work on X; autosome translocations in human and mouse suggested failure of stabilization of XCI in autosomal material, so that genes are reactivated, but resistance of autosomal genes to the original silencing is not excluded. The accumulation of L1s on the X-chromosome may have resulted from reduced recombination or late replication. Whether L1s are part of the mechanism of XCI or a result of it remains enigmatic.
\end{abstract}

Copyright (c) 2006 Mary F. Lyon. This is an open access article distributed under the Creative Commons Attribution License, which permits unrestricted use, distribution, and reproduction in any medium, provided the original work is properly cited.

\section{INTRODUCTION}

At an early stage in the discovery of X-chromosome inactivation $(\mathrm{XCI})$, the phenomena seen in mouse $\mathrm{X}$; autosome translocations provided important clues. The first relevant observation that led to the discovery of XCI was that female mice heterozygous for X-linked colour genes showed characteristic patterns of colour variegation in their coats different from the patterns produced by autosomal colour genes [1]. Similar variegated coat colour patterns occurred in females heterozygous for X; autosome translocations when the translocated autosomal segment included loci of coat colour genes [2]. This was attributed to the travel of the inactivating signal from the X-chromosome into the attached autosome. However, the inactivation of colour genes occurred in only one of the two segments into which the autosome was broken. This led to the concept of the X-inactivation centre (XIC) on the X-chromosome, from which the silencing signal spread in a cis-limited manner along the chromosome $[3,4]$. Only X-chromosomal or autosomal segments in physical continuity with the XIC underwent inactivation. Another feature seen in X; autosome translocations was that the autosomal segment involved did not undergo complete inactivation. Genes distant from the translocation break showed less variegation, and therefore less inactivation, than genes nearer to the break [4]. It appeared that the silencing signal travelled less successfully in autosomal than in X-chromosomal chromatin.

The concept of the X-inactivation centre has led to major advances. The XIST/Xist gene (XIST is the human gene symbol and Xist the mouse gene symbol) cloned from the XIC has been shown to be necessary and sufficient for the initiation of XCI in the early embryo $[5,6]$. Xist codes for an untranslated mRNA which coats the inactive X-chromosome (Xi) [7] and initiates gene silencing. This is followed by a process of stabilization of the inactive state, which is independent of Xist and involves a complex series of chromatin changes, including histone modifications and methylation of $\mathrm{CpG}$ islands (reviewed in [8]). The Xi becomes late replicating, its histones $\mathrm{H} 3$ and $\mathrm{H} 4$ are hypoacetylated, histone $\mathrm{H} 3$ is methylated at lysines $\mathrm{K} 9$ and $\mathrm{K} 27$, its CpG islands are methylated, and it is associated with the variant histone macro-H2A.1. These are the so-called hallmarks of XCI.

Thus, much is known concerning the initiation of XCI but the basis for the apparently less successful travel of the silencing in autosomal than in X-chromosomal chromatin remains enigmatic. Riggs $[9,10]$ suggested the existence of "way stations" or "boosters" along the X-chromosome which enhanced the spread of XCI. I suggested that interspersed repeat elements, specifically LINE1s, were candidates for the boosters $[11,12]$. This paper provides a review of the evidence for and against this suggestion. 


\section{EVIDENCE FOR CANDIDATURE OF LINE1s AS BOOSTER ELEMENTS}

\section{Richness of LINE1s on the X-chromosome}

Although XCI is incomplete in autosomal material, it seems, from many human $\mathrm{X}$; autosome translocations and from mouse transgenes, that it can travel to some extent in any autosome. Therefore, to be candidates for boosters, elements must be present throughout the genome but must be particularly dense on the X-chromosome. The mouse and human X-chromosomes were first described as being rich in L1s on the basis of FISH (fluorescent in situ hybridization) evidence with antibodies to L1s [13, 14]. This was later extended to other species $[15,16]$. In the fruit bat Carollia, an $\mathrm{X}$; autosome translocation forms part of the normal karyotype. Parish et al [17] found that the X-chromosomal part of the translocation was rich in L1s but the autosomal part was no richer than other autosomes. Thus, the data suggest that richness in L1s is a common feature of mammalian Xchromosomes.

Data accruing from the human genome project have yielded further detailed information on the distribution of L1s along the human X-chromosome. Bailey et al [18] compared the human $\mathrm{X}$-chromosome with several autosomes and found the X-chromosome to be indeed rich in L1s, having $26 \%$ L1s compared with only $13 \%$ in autosomal DNA. On the human X-chromosome, some genes escape XCI and remain active on the $\mathrm{Xi}$. These escaping genes tend to be clustered in certain regions, particularly in the distal part of the short arm, Xp. Bailey et al found that the concentration of L1s in this distal part of Xp was lower than in the vicinity of the XIC. They also studied the age of the L1s and found that they were predominantly of a younger age, dating from the time in evolution of the eutherian radiation. Ross et al [19] studied the complete annotated sequence of the human Xchromosome and were able to provide further detail on the distribution of L1s. They confirmed that the X-chromosome was indeed rich in L1s, having $29 \%$ L1s compared with a genome average of $17 \%$. The density of L1s was high around the XIC but the XIST gene itself lay in a $60 \mathrm{~kb}$ region not rich in L1s. Distal Xp, where there are many genes escaping XCI, had an L1 density not greater than that in autosomes. Graves and Watson [20] had studied the evolutionary history of the $\mathrm{X}$-chromosome and divided it into an older X-conserved region (XCR), which had evolved from an autosome at an early stage, and a region more recently added from an autosome, the $\mathrm{X}$-added region, XAR. These regions were further divided into five subregions according to their evolutionary age [21]. Ross et al found that the density of L1s in the various regions was strongly correlated with evolutionary age, being highest in the evolutionarily oldest regions.

Thus, these data are compatible with L1s having a role as boosters in XCI. However, they do not reveal whether L1s are part of the mechanism of XCI or whether the density of L1s is a consequence of XCI.

Evidence that appears to conflict with the data of Bailey et $\mathrm{al}$ and Ross et al comes from the work of Ke and Collins [22]. They studied the density of L1s at points from $1 \mathrm{~kb}$ to $100 \mathrm{~kb}$ upstream or downstream of 19 human genes that escaped $\mathrm{XCI}$ and 73 normally inactivated genes and found no significant differences. They did, however, find a lower density of CpG islands around escaping genes. This work in turn was contradicted by Carrel and Willard [23] who studied a much larger number of escaping and nonescaping genes, and found no difference in $\mathrm{CpG}$ island content but, like Ross et al, found that regions with many escaping genes did have a lower L1 content. Two groups have studied chicken transgenes introduced into the mouse X-chromosome, to determine if they would be subject to XCI, given that they are unlikely to contain recognition sequences important for XCI (such as young L1s that are absent in the chicken genome). Riggs [24] introduced a chicken transgene into the mouse Hprt locus. The transgene had complete flanking sequences but no L1s and yet it was inactivated when on the Xi. However, Goldman et al $[25,26]$ introduced a larger construct, consisting of 11 copies of the chicken $17 \mathrm{~kb}$ transferrin gene (187 kb in all), into the mouse $\mathrm{X}$-chromosome and the transgene escaped inactivation. This is consistent with L1s acting over relatively large distances.

As in the human, data from the mouse genome sequencing project have confirmed that the mouse X-chromosome is rich in L1s having $28.5 \% \mathrm{~L} 1 \mathrm{~s}$ compared with $14.6 \%$ in autosomes [27]. The mouse Y-chromosome also is rich in L1s. Tsuchiya et al [28] studied a region of the human X, Xp11.2, that contains a domain of escaping genes and compared it with a conserved region in the mouse, which has only one escaping gene. They found no association of L1 density with escape from XCI, except that L1 density was reduced in the $S M C X / S m c x$ gene region. In contrast, the density of long terminal repeats (LTRs) was decreased in the human escaping region.

\section{Recent data on X; autosome translocations}

Incomplete XCI in autosomal segments attached to the Xchromosome in $\mathrm{X}$; autosome translocations could have various causes. The silencing signal could fail to travel in autosomal chromatin, or autosomal genes might be resistant to the signal, or the stabilization of XCI might fail so that genes undergo reactivation. It is difficult to tell these causes apart in specific cases, but some clues are available. In some cases, genes at a long distance from the translocation break in the autosome are inactivated, while some other interstitial genes are not. In such a case, the escape of the interstitial genes from XCI must be either due to resistance to the original silencing signal or to initial silencing followed by reactivation. To distinguish between these two possibilities requires observations at different times. For example, observations might be made of mouse embryos and liveborn young of various ages, or cultured cells might be followed over different passages. In early work on the mouse, there were several translocations involving chromosome 7 and the coat colour genes albino, $c$, and pinkeye, $p$ [29]. Both the $c$ and $p$ genes showed variegation, resulting from the inactivation of the wild-type alleles in the translocated segment, but the level of the variegation and hence of the inactivation 
varied from one translocation to another. Hence, incomplete $\mathrm{XCI}$ of the two colour genes cannot have been solely due to their resistance to the silencing signal or the stabilizing mechanism. The degree of travel of the signals into the autosome must have been at least partly responsible, and this is confirmed by the fact that variegation was lower with greater distance of the genes concerned from the XIC. Evidence that reactivation was also involved was provided by Cattanach [30] who showed that animals with inactivation of the wild-type allele of the albino gene due to the translocation Is $(\operatorname{In} 7 ; \mathrm{X}) 1 \mathrm{Ct}$ darkened with age, as the wild-type allele regained expression. The distribution of the pigmented cells was such that they could not have arisen by movement of pigmented cells around the body, but must have arisen by change in expression of pigment genes. Thus, in this group of translocations, it appears that both failure of travel of the signal and failure of stabilization were involved.

Recently there has been further work with mouse and human translocations. In the mouse, Duthie et al [31] studied two translocations resulting in variegation for coat colour genes, $\mathrm{T}(4 ; \mathrm{X}) 37 \mathrm{H}$ which gives variegation for the gene brown, $b$, and Is(In7;X)1Ct (previously studied by Cattanach) which gives variegation for albino, $c$, pink-eye, $p$, and ruby2, ru2. They found only limited coating of the autosomal material with Xist RNA and variation from cell to cell. Similarly, the spread of hypoacetylation of histone $\mathrm{H} 4$ into the autosome was limited and variable. In the X-chromosome itself, Xist RNA was restricted to light G-bands. These results are consistent with initial spread of Xist RNA to a point beyond that of the inactivated genes, followed by the failure of the stabilization mechanism so that hallmarks of XCI, such as hypoacetylation of histones, did not appear, and in some cells genes were reactivated. Resistance of some genes to the original silencing signal is also possible. As Cattanach [30] had previously shown the reactivation of the wild-type allele of the $c$ gene in $\mathrm{Is}(\operatorname{In} 7 ; \mathrm{X}) 1 \mathrm{Ct}$, it is tempting to attribute all the effects seen to reactivation, but in fact the failure of travel of the silencing signal and the resistance of some genes to it are also possible.

Recent results with human $\mathrm{X}$; autosome translocations have also suggested the occurrence of reactivation of autosomal material. These studies have used unbalanced translocation products. In individuals with balanced X; autosome translocations the translocated X-chromosome is typically the active one $(\mathrm{Xa})$ in all cells. Inactivation of the translocated $\mathrm{X}$ leads to genetic imbalance, because of the inactivation of the attached autosome and failure of inactivation of the X-chromosome segment lacking the XIC. Cells with the translocated $\mathrm{X}$-chromosome as the $\mathrm{Xi}$ are thus selected against. Conversely, in an unbalanced translocation, inactivation of the translocated segment tends to restore genetic balance and is selected. White et al [32] studied a female with an unbalanced X; 4 translocation and a normal phenotype, suggesting that most of the excess chromosone 4 segment was inactive. They found that 14/20 genes and expressed sequence tags (ESTs) were inactivated and $6 / 20$ were expressed. The inactivated genes were distributed widely along the autosomal segment, indicating that the inactivating signal had travelled a long distance along the segment, over $100 \mathrm{Mb}$. Five of the six expressed sequences were interstitial, having inactivated sequences on both sides. These results were consistent either with the resistance of these genes to the original silencing signal or with their reactivation.

A similar picture consistent with either resistance to silencing, or with reactivation, or with both has emerged from other studies with human $\mathrm{X}$; autosome translocations, in which various hallmarks of XCI have been studied. Keohane et al [33] found no spread into the autosome of XIST RNA, hypoacetylation, or late replication in two unbalanced translocations, but others obtained different results. Sharp et al [34] studied five unbalanced translocations, one of which had also been used by Keohane et al and found considerable variation in the spread of XCI. The overall picture was of discontinuous spread of XCI, with some interstitial genes not inactivated. Hallmarks of XCI, including hypoacetylation of histones, late replication, and $\mathrm{CpG}$ island methylation, varied from case to case and from cell to cell. In contrast to the results of Keohane et al, they found some spreading of XCI into the autosome in the case also studied by these authors. Hall et al [35] studied two unbalanced translocations in which the relatively normal phenotype suggested considerable gene inactivation. In both translocations, only part of the autosomal segment was coated with XIST RNA. In one, the autosome showed hypoacetylation, and in both, late replication varied from cell to cell. The autosomal segments seemed to show more hallmarks of XCI than was indicated by the XIST RNA coverage, and the authors suggested that the XIST RNA had originally extended further. The work was done with cultured cells, and at earlier passages of the same cells, complete late replication of the autosomal segment had been observed, whereas it was now only partial, suggesting a loss of late replication with time. Failure of stabilization of inactivation seemed a better explanation of the effects seen than resistance to the original silencing signal.

Thus, the evidence as a whole from X; autosome translocations is that incomplete XCI in autosomal material involves a combination of factors. The early work on mouse translocations indicated incomplete travel of the original silencing signal in different translocations. The more recent data suggest that, in addition, there may be resistance of some genes to the silencing signal, and also failure of the stabilizing signal, leading to reactivation. The possibility of reactivation would fit with the earlier work of Cattanach [30] on the reactivation of the albino gene with age in the insertion Is1Ct. It is also consistent with the studies of Hall et al [36] who introduced an XIST transgene into an autosome in human cultured cells, and found diminution of signs of XCI with time in culture. The general picture at present is that both the original silencing and also the stabilizing mechanism are liable to failure in autosomal material.

\section{Possible mechanisms of action of LINE1s}

When the Xist gene is introduced as a transgene into an autosome, effects similar to those in $\mathrm{X}$; autosome translocations 
are seen. The Xist RNA coats the autosome, but not completely, and the genes are silenced, but those at a distance from the transgene may escape silencing [37]. Wutz and Jaenisch [38] constructed an inducible Xist cDNA transgene and introduced it into an autosome in mouse embryonic stem (ES) cells. In undifferentiated ES cells, the Xist RNA travelled along the chromosome and brought about silencing of genes. This silencing was not accompanied by hallmarks of XCI and was reversed when the agent inducing the cDNA expression was removed. When the ES cells were allowed to differentiate in the presence of Xist cDNA, hallmarks of $\mathrm{XCI}$ appeared and the silencing became irreversible and independent of expression of Xist by the inducing agent. Thus, there is some developmental factor, unidentified at present, required for the start of the stabilizing process. The timing of travel of Xist RNA is hence very important. If this RNA is not in place when the developmental factor initiates stabilization, the process will fail. It is not yet known how long is required for the process of coating of the chromosome with Xist RNA. However, data of Latham [39] and of Huynh and Lee [40], that in early mouse embryos X-linked genes at a distance from the XIC remain active at later stages than those near the XIC, suggest that it is quite a slow process. Possibly, L1s could affect the speed of travel of Xist RNA. Another possibility is that they could promote the attachment of Xist RNA to the chromatin, and hence could aid in the stabilization.

The process of stabilization might also be affected in other ways. L1s might affect the binding of histone-modifying enzymes or might affect $\mathrm{CpG}$ island methylation. Hansen [41] studied methylation of L1s in females suffering from the autosomal recessive ICF syndrome (immunodeficiency, centromeric instability, and facial anomalies) caused by the deficiency of the DNMT3B de novo DNA methyltransferase gene. These females showed normal methylation of $\mathrm{CpG}$ islands and L1s on autosomes and the active $\mathrm{X}$, but reduced methylation of both L1s and CpG islands on the inactive $\mathrm{X}$-chromosome. This indicated that L1s and CpG islands on the inactive $\mathrm{X}$ are methylated by DNMT3B, and that autosomal and active X L1s are methylated by some other enzyme. Hansen concluded that X-chromosomal L1s are probably unmethylated at the time of initiation of XCI in the early embryo. This is consistent with them having a role in XCI. Another possibility is that L1s tend to change the state of chromatin towards the heterochromatic state and this again might tend to assist stabilization of XCI [42].

An interesting point is that Allen et al [43] found an excess of L1s in the flanking regions of autosomal monoallelically expressed genes, both imprinted genes and randomly monoallelically expressed genes. In human and mouse, the L1s were primarily of an evolutionarily young, speciesspecific type, suggesting that they had accumulated after the genes became monoallelic, and that the genes concerned had developed a strategy for monoallelic expression involving L1s after first becoming monoallelic. As with the X-chromosome, the role of these L1s is not clear.

\section{MECHANISM OF ACCUMULATION OF LINE1s ON THE X-CHROMOSOME}

Consideration of the possible means of accumulation of L1s on the X-chromosome might yield some clues as to whether this accumulation is a part of the mechanism of XCI or is a result of it. One possibility is that L1s have accumulated as a result of reduction in recombination on the $\mathrm{X}$-chromosome as the $\mathrm{X}$ - and Y-chromosomes have differentiated. The Ychromosome is thought to have differentiated from the $\mathrm{X}$ chromosome by a series of inversions $[19,44]$. Each inversion will have led to an absence in males of recombination in the inverted segment. This will have been followed by the degeneration of the isolated segment of the Y-chromosome. It has been suggested that the level of L1s in mammalian genomes is a result of competition between insertion and excision, and excision is thought to involve recombination. Thus, as recombination on the $\mathrm{X}$-chromosome decreased in evolution, L1s would accumulate $[16,45]$. In the Drosophila species $D$ miranda retrotransposons were seen to accumulate during the evolution of a neo-Y chromosome [42], although they did not accumulate on the X. Another possibility is that late replication of the X-chromosome when inactive might lead to an accumulation of L1s [16]. Thus, accumulation of L1s might be due to differentiation of the Xand Y-chromosomes in evolution and in this sense incidental to XCI. It should be noted, however, that the age of the L1s dates them from the time of the eutherian radiation [18], well after the time of the differentiation of the X- and Ychromosomes. Even if the accumulation of L1s is incidental to XCI, they could still be part of the mechanism. There could be a self-sustaining system. It is possible that as the $\mathrm{X}$ - and Y-chromosomes differentiated, L1s accumulated and this favoured the spread of XCI, which presumably began at the XIC. Further accumulation of L1s in evolution could then have been selected.

\section{CONCLUSION}

At present, there is good evidence for an accumulation of L1s on the human X-chromosome in such a distribution that they could fulfil the function of booster elements in XCI. Whether or not they indeed have such a function is less clear. Information from the sequence of the human Xchromosome showing that regions with many escaping genes have a lower density of L1s is very provocative. In addition to the more limited coverage of Tsuchiya et al [28], it would be very valuable to have similar data on the mouse Xchromosome, which has been much rearranged in evolution and where there are fewer escaping genes.

\section{REFERENCES}

[1] Lyon MF. Gene action in the $X$-chromosome of the mouse (Mus musculus L.). Nature. 1961;190(4773):372-373.

[2] Russell LB, Bangham JW. Variegated-type position effects in the mouse. Genetics. 1961;46(5):509-525. 
[3] Lyon MF. Cytogenetics, discussion. In: Proceedings of the Second International Conference on Congenital Malformations; July 1963; New York, NY. 67-68.

[4] Russell LB. Mammalian X-chromosome action: inactivation limited in spread and region of origin. Science. 1963;140:976978.

[5] Penny GD, Kay GF, Sheardown SA, Rastan S, Brockdorff N. Requirement for Xist in X chromosome inactivation. Nature. 1996;379(6561):131-137.

[6] Wutz A, Jaenisch R. A shift from reversible to irreversible X inactivation is triggered during ES cell differentiation. Molecular Cell. 2000;5(4):695-705.

[7] Clemson CM, McNeil JA, Willard HF, Lawrence JB. XIST RNA paints the inactive $\mathrm{X}$ chromosome at interphase: evidence for a novel RNA involved in nuclear/chromosome structure. The Journal of Cell Biology. 1996;132(3):259-275.

[8] Heard E. Recent advances in X-chromosome inactivation. Current Opinion in Cell Biology. 2004;16(3):247-255.

[9] Gartler SM, Riggs AD. Mammalian X-chromosome inactivation. Annual Review of Genetics. 1983;17:155-190.

[10] Riggs AD. Marsupials and mechanisms of X-chromosome inactivation. Australian Journal of Zoology. 1990;37(2):419-441.

[11] Lyon MF. Epigenetic inheritance in mammals. Trends in Genetics. 1993;9(4):123-128.

[12] Lyon MF. X-chromosome inactivation: a repeat hypothesis. Cytogenetics and Cell Genetics. 1998;80(1-4):133-137.

[13] Boyle AL, Ballard SG, Ward DC. Differential distribution of long and short interspersed element sequences in the mouse genome: chromosome karyotyping by fluorescence in situ hybridization. Proceedings of the National Academy of Sciences of the United States of America. 1990;87(19):7757-7761.

[14] Korenberg JR, Rykowski MC. Human genome organization: $\mathrm{Alu}$, lines, and the molecular structure of metaphase chromosome bands. Cell. 1988;53(3):391-400.

[15] Baker RJ, Wichman HA. Retrotransposon Mys is concentrated on the sex chromosomes: implications for copy number containment. Evolution. 1990;44(8):2083-2088.

[16] Wichman HA, Van den Bussche RA, Hamilton MJ, Baker RJ. Transposable elements and the evolution of genome organization in mammals. Genetica. 1992;86(1-3):287-293.

[17] Parish DA, Vise P, Wichman HA, Bull JJ, Baker RJ. Distribution of LINEs and other repetitive elements in the karyotype of the bat Carollia: implications for X-chromosome inactivation. Cytogenetic and Genome Research. 2002;96(1-4):191-197.

[18] Bailey JA, Carrel L, Chakravarti A, Eichler EE. Molecular evidence for a relationship between LINE-1 elements and X chromosome inactivation: the Lyon repeat hypothesis. Proceedings of the National Academy of Sciences of the United States of America. 2000;97(12):6634-6639.

[19] Ross MT, Grafham DV, Coffey AJ, et al. The DNA sequence of the human X chromosome. Nature. 2005;434(7031):325-337.

[20] Graves JAM, Watson JM. Mammalian sex chromosomes: evolution of organization and function. Chromosoma. 1991; 101(2):63-68.

[21] Lahn BT, Page DC. Four evolutionary strata on the human X chromosome. Science. 1999;286(5441):964-967.

[22] Ke X, Collins A. CpG islands in human X-inactivation. Annals of Human Genetics. 2003;67(3):242-249.

[23] Carrel L, Willard HF. X-inactivation profile reveals extensive variability in X-linked gene expression in females. Nature. 2005;434(7031):400-404.
[24] Riggs AD. X chromosome inactivation, differentiation, and DNA methylation revisited, with a tribute to Susumu Ohno. Cytogenetic and Genome Research. 2002;99(1-4):17-24.

[25] Goldman MA, Stokes KR, Idzerda RL, et al. A chicken transferrin gene in transgenic mice escapes X-chromosome inactivation. Science. 1987;236(4801):593-595.

[26] Goldman MA, Reeves PS, Wirth CM, et al. Comparative methylation analysis of murine transgenes that undergo or escape X-chromosome inactivation. Chromosome Research. 1998;6(5):397-404.

[27] Waterston RH, Lindblad-Toh K, Birney E, et al. Initial sequencing and comparative analysis of the mouse genome. $\mathrm{Na}$ ture. 2002;420(6915):520-562.

[28] Tsuchiya KD, Greally JM, Yi Y, Noel KP, Truong JP, Disteche $\mathrm{CM}$. Comparative sequence and $\mathrm{X}$-inactivation analyses of a domain of escape in human Xp11.2 and the conserved segment in mouse. Genome Research. 2004;14(7):1275-1284.

[29] Russell LB, Montgomery CS. Comparative studies on Xautosome translocations in the mouse. II. Inactivation of autosomal loci, segregation, and mapping of autosomal breakpoints in five T(X;1)'S. Genetics. 1970;64(2):281-312.

[30] Cattanach BM. Position effect variegation in the mouse. Genetical Research. 1974;23(3):291-306.

[31] Duthie SM, Nesterova TB, Formstone EJ, et al. Xist RNA exhibits a banded localization on the inactive X chromosome and is excluded from autosomal material in cis. Human Molecular Genetics. 1999;8(2):195-204.

[32] White WM, Willard HF, Van Dyke DL, Wolff DJ. The spreading of $\mathrm{X}$ inactivation into autosomal material of an X;autosome translocation: evidence for a difference between autosomal and X-chromosomal DNA. The American journal of Human Genetics. 1998;63(1):20-28.

[33] Keohane AM, Barlow AL, Waters J, Bourn D, Turner BM. H4 acetylation, XIST RNA and replication timing are coincident and define X;autosome boundaries in two abnormal X chromosomes. Human Molecular Genetics. 1999;8(2):377-383.

[34] Sharp AJ, Spotswood HT, Robinson DO, Turner BM, Jacobs PA. Molecular and cytogenetic analysis of the spreading of $\mathrm{X}$ inactivation in X;autosome translocations. Human Molecular Genetics. 2002;11(25):3145-3156.

[35] Hall LL, Clemson CM, Byron M, Wydner K, Lawrence JB. Unbalanced X;autosome translocations provide evidence for sequence specificity in the association of XIST RNA with chromatin. Human Molecular Genetics. 2002;11(25):3157-3165.

[36] Hall LL, Byron M, Sakai K, Carrel L, Willard HF, Lawrence JB. An ectopic human XIST gene can induce chromosome inactivation in postdifferentiation human HT-1080 cells. Proceedings of the National Academy of Sciences of the United States of America. 2002;99(13):8677-8682.

[37] Lee JT, Jaenisch R. Long-range cis effects of ectopic Xinactivation centres on a mouse autosome. Nature. 1997;386 (6622):275-279.

[38] Wutz A, Jaenisch R. A shift from reversible to irreversible X inactivation is triggered during ES cell differentiation. Molecular Cell. 2000;5(4):695-705.

[39] Latham KE. X chromosome imprinting and inactivation in preimplantation mammalian embryos. Trends in Genetics. 2005;21(2):120-127.

[40] Huynh KD, Lee JT. Inheritance of a pre-inactivated paternal X chromosome in early mouse embryos. Nature. 2003; 426(6968):857-862. 
[41] Hansen RS. X inactivation-specific methylation of LINE-1 elements by DNMT3B: implications for the Lyon repeat hypothesis. Human Molecular Genetics. 2003;12(19):2559-2567.

[42] Steinemann M, Steinemann S. Enigma of Y chromosome degeneration: neo- $Y$ and neo-X chromosomes of Drosophila miranda a model for sex chromosome evolution. Genetica. 1998;102-103(1):409-420.

[43] Allen E, Horvath S, Tong F, et al. High concentrations of long interspersed nuclear element sequence distinguish monoallelically expressed genes. Proceedings of the National Academy of Sciences of the United States of America. 2003;100(17):99409945.

[44] Graves JAM. The origin and function of the mammalian Y chromosome and Y-borne genes - an evolving understanding. BioEssays. 1995;17(4):311-320.

[45] Langley CH, Montgomery E, Hudson R, Kaplan N, Charlesworth B. On the role of unequal exchange in the containment of transposable element copy number. Genetical Research. 1988;52(3):223-235. 

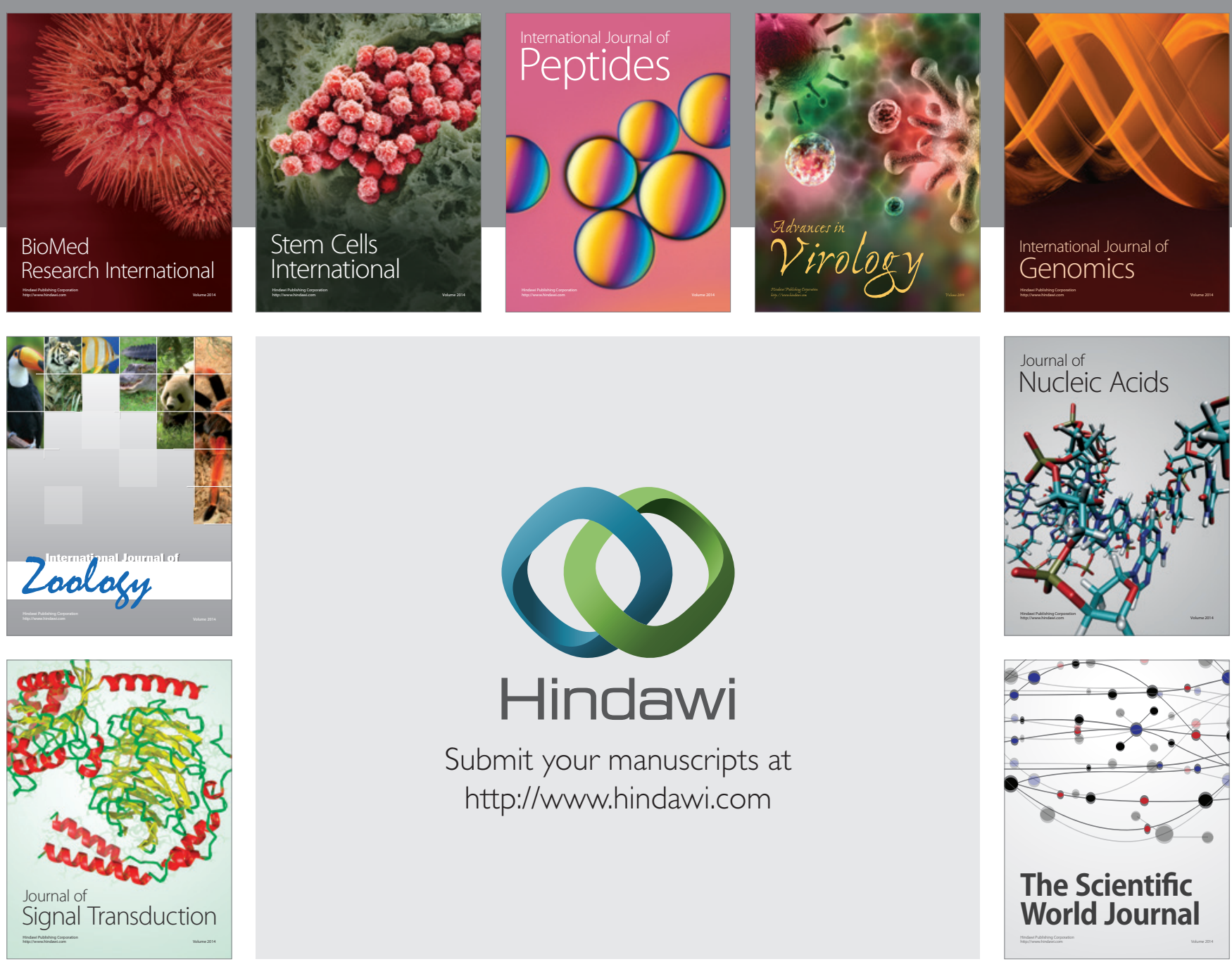

Submit your manuscripts at

http://www.hindawi.com
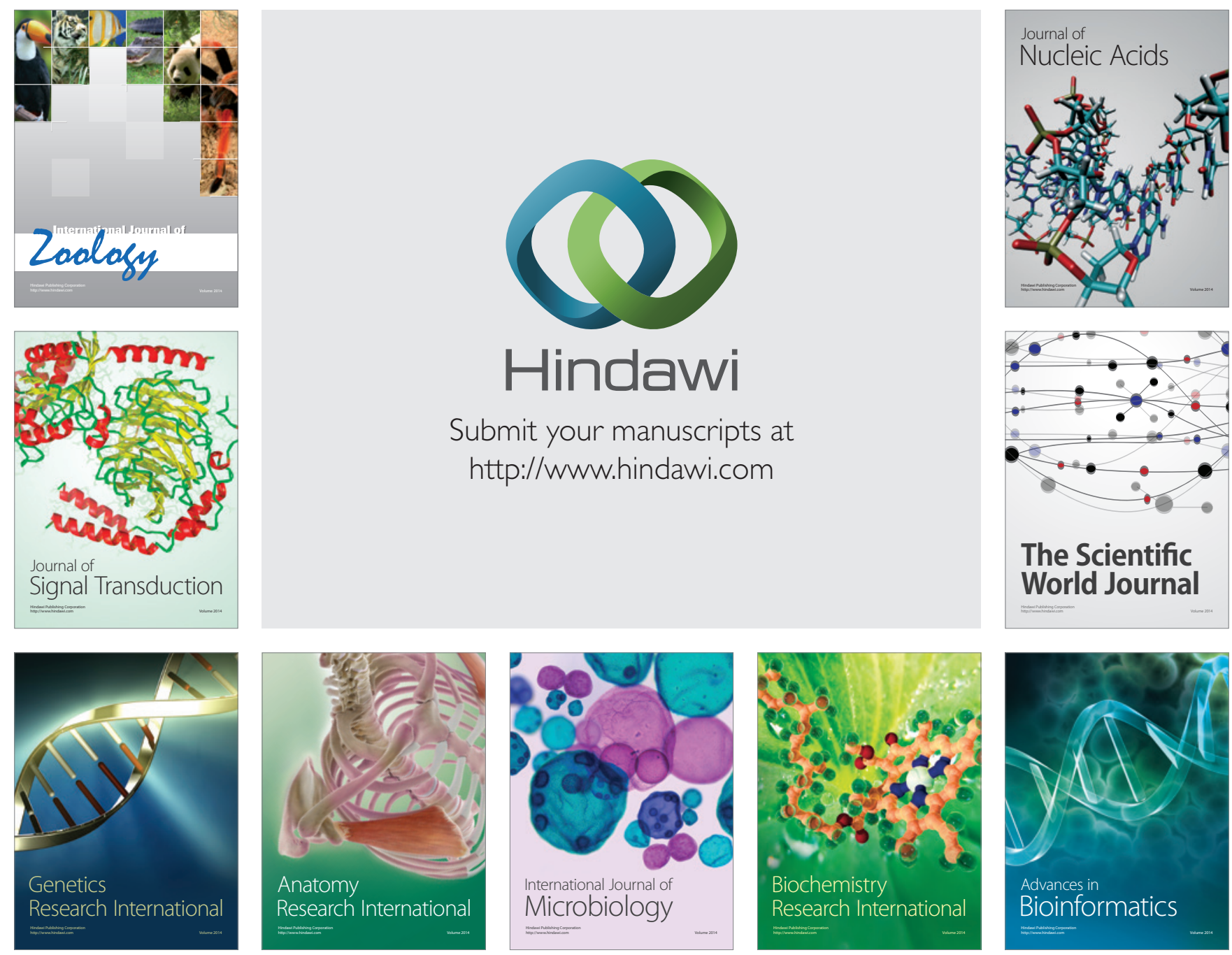

The Scientific World Journal
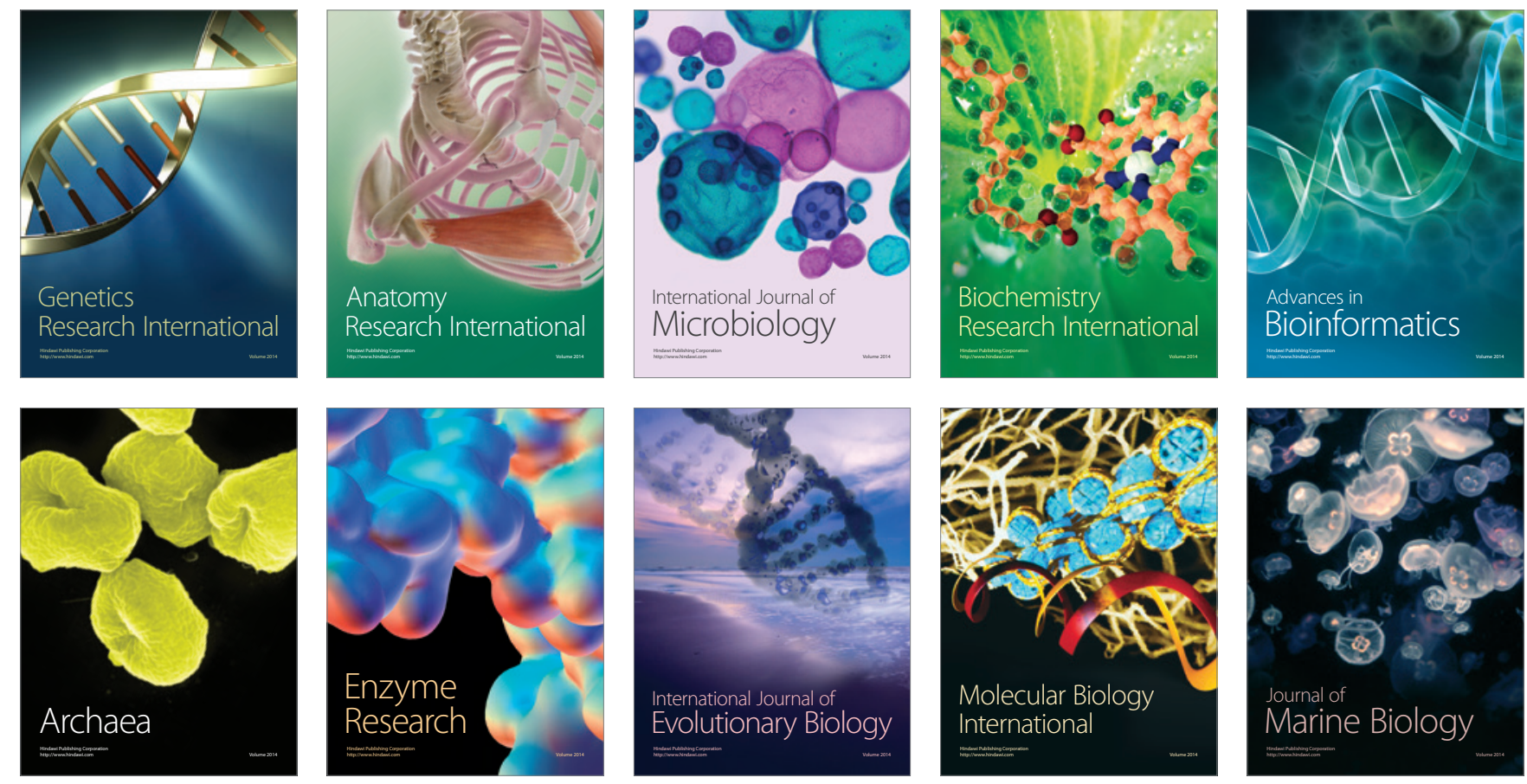